\title{
Precise Three-Dimensional Morphology of the Male Anterior Anorectum Reconstructed From Large Serial Histologic Sections: A Cadaveric Study
}

\section{$\operatorname{AUTHOR}(\mathrm{S}):$}

Okada, Tomoaki; Hasegawa, Suguru; Nakamura, Tatsuro; Hida, Koya; Kawada, Kenji; Takai, Akihiro; Shimokawa, Tetsuya; Matsuda, Seiji; Shinohara, Hisashi; Sakai, Yoshiharu

\section{CITATION:}

Okada, Tomoaki ...[et al]. Precise Three-Dimensional Morphology of the Male Anterior Anorectum Reconstructed From Large Serial Histologic Sections: A Cadaveric Study. Diseases of the colon and rectum 2019, 62(10): 1238-1247

\section{ISSUE DATE:}

2019-10

URL:

http://hdl.handle.net/2433/259293

\section{RIGHT:}

(c) 2019 Wolters Kluwer Health | Lippincott Williams \& Wilkins. This is a non-final version of an article published in final form in Okada, T., et al. Precise Three-Dimensional Morphology of the Male Anterior Anorectum Reconstructed From Large Serial Histologic Sections: A Cadaveric Study, Diseases of the Colon \& Rectum. 2019; 62(10): 1238-1247; この こ論文 は出版社版でありません。引用の際には出版社版をご確認ご利用ください。; This is not the published version. Please cite only the published version. 


\section{Dynamic Article}

2 Precise three-dimensional morphology of the male anterior anorectum reconstructed from large

3 serial histological sections: a cadaveric study

4

5 Running title: 3D morphology of male anterior anorectum

6

7 Tomoaki Okada, M.D. ${ }^{1}$, Suguru Hasegawa, M.D., Ph.D., FACS ${ }^{2}$, Tatsuro Nakamura, M.D. ${ }^{1}$,

8 Koya Hida, M.D., Ph.D. ${ }^{1}$, Kenji Kawada, M.D., Ph.D. ${ }^{1}$, Akihiro Takai, M.D., Ph.D. ${ }^{3}$, Tetsuya

9 Shimokawa, D.V.M., Ph.D. ${ }^{4}$, Seiji Matsuda, M.D., Ph.D. ${ }^{4}$, Hisashi Shinohara, M.D., Ph.D. ${ }^{5}$

10 Yoshiharu Sakai, M.D., Ph.D., FACS ${ }^{1}$

11 1. Department of Surgery, Kyoto University Graduate School of Medicine, Kyoto, Japan

12 2. Department of Gastroenterological Surgery, Fukuoka University Faculty of Medicine,

$13 \quad$ Fukuoka, Japan

14 3. Department of HBP and Breast Surgery, Ehime University Graduate School of Medicine,

15 Ehime, Japan

16 4. Department of Anatomy and Embryology, Ehime University Graduate School of Medicine,

17 Ehime, Japan 
1 5. Department of Surgery, Hyogo College of Medicine, Hyogo, Japan

2

3 Corresponding author: Tomoaki Okada, MD

4 Department of Surgery, Kyoto University Graduate School of Medicine

554 Shogoin Kawahara-cho, Sakyo-ku, Kyoto 606-8507, Japan

6 Tel: (+81)-75-366-7595, Fax: (+81)-75-366-7642

$7 \quad$ Email: t.okada@kuhp.kyoto-u.ac.jp

8

$9 \quad$ Financial disclosure: None reported.

11 Supplemental digital contents: This article contains three supplemental digital content files.

13 Funding/Support: This work was supported by the Japan Society for the Promotion of Science

14 (KAKENHI numbers: 16H05399 and 16K10538).

16 Previous presentation: European Society of Coloproctology, Niece, France, September 26-

$17 \quad 28,2018$ 
1 Text word count: 3078/3000; Abstract word count: 300/300

2

\section{Authorship contribution}

4 Study conception and design: Okada, Hasegawa, Shimokawa, Matsuda, Shinohara, and Sakai

5 Acquisition of data: Okada, Nakamura, Shimokawa, and Takai

6 Analysis and interpretation of data: Okada, Hasegawa, Hida, Kawada, Sakai

7 Drafting of manuscript: Okada and Hasegawa

8 Critical revision for intellectual content: All authors

$9 \quad$ Final approval of the manuscript: All authors

10 Agreement to be accountable for all aspects of the work in ensuring that questions related to

11 the accuracy or integrity of any part of the work are appropriately investigated and resolved:

12 All authors

13

14 Category: Colorectal/Anal Neoplasia 


\section{$1 \quad$ Abstract}

2 Background: Deep anatomical knowledge of the male anterior anorectum is important to

3 avoid urethral injury and rectal perforation in intersphincteric resection or abdominoperineal

4 resection for very low rectal cancer. However, its structure is difficult to understand because

5 the anorectum, muscles, and urogenital organs are complicatedly and three-dimensionally

6 arranged.

7 Objective: To revisit the anatomical information of the male anterior anorectum for

8 intersphincteric resection and abdominoperineal resection with a focus on the spatial muscular

9 morphology.

10 Design: Descriptive cadaveric study.

11 Setting: Ehime and Kyoto University.

12 Patients: Tissue specimens from nine male cadavers.

13 Main Outcome Measures: Specimens around the anterior anorectum were serially sectioned

14 in the horizontal, sagittal, or frontal plane; large semi-serial histological sections were created

15 at $250-\mu \mathrm{m}$ intervals. The series were stained with Elastica van Gieson, and some sections from

16 the series were studied by immunohistochemistry to detect smooth and striated muscles. Two

17 series were digitalized and reconstructed three-dimensionally. 
1 Results: Two regions without a clear anatomical border were elucidated: (i) the anterior region

2 of the external anal sphincter, where the external anal sphincter, bulbospongiosus muscle, and

3 superficial transverse perineal muscle were intertwined; and (ii) rectourethralis muscle, where

4 the smooth muscle of the longitudinal muscle continuously extended to the posteroinferior area

5 of the urethra, which became closest to the anorectum at the prostatic apex level. Tight

6 connection between the striated and smooth muscles was identified at the anterior part of the

7 upper external anal sphincter and anterolateral part of the puborectalis muscle level.

8 Limitation: This study involved a small sample size of elderly cadavers.

9 Conclusion: This study clarified the precise spatial relationship between smooth and striated

10 muscles. The detailed anatomical findings will contribute more accurate step-by-step anterior

11 dissection in intersphincteric resection and abdominoperineal resection, especially with the

12 transanal approach, which can magnify the muscle fiber direction and contraction of striated muscle by electrostimulation. 


\section{$1 \quad$ Introduction}

2 Low anterior resection with intersphincteric resection or abdominoperineal resection is

3 performed for very low rectal cancer. Negative circumferential resection margins and negative

4 distal margins reduce the risk of local recurrence. However, abdominoperineal resection has a

5 high rate of positive surgical margin and rectal perforation compared with sphincter-preserving

6 surgery. ${ }^{1,2}$ One of the causes of this high rate is the difficulty of determining the dissection

7 plane around the anterior anorectum, which contains the perineal body or the rectourethralis

8 muscle (RUM). ${ }^{3}$ Recently, transanal total mesorectal excision has been gradually gaining

9 popularity as a novel approach for low rectal cancer. ${ }^{4}$ Although magnified endoscopic

10 visualization around the anorectum and the pelvic floor can be obtained, urethral or rectal injury

11 in male patients has been recognized commonly by colorectal surgeons as one of the

12 devastating complications. ${ }^{4,5}$ Thus, deep anatomical knowledge of the anterior anorectum,

13 especially of men, is important to avoid urethral injury, rectal perforation, and positive surgical

14 margins in abdominoperineal resection and intersphincteric resection.

15 The anorectum mainly consists of smooth and striated muscles; smooth muscles are the circular

16 muscle layer $(\mathrm{CM})$ and the longitudinal muscle layer (LM) of the rectal muscularis propria,

17 and striated muscles are the external anal sphincter (EAS) and the puborectalis muscle (PRM), 
1 which is the innermost part of the levator ani muscle. ${ }^{6-8}$ The detailed knowledge of the

2 relationship between these striated and smooth muscles is important for abdominoperineal

3 resection and intersphincteric resection, ${ }^{3,6}$ because these two-type muscles are distinguishable

4 by their color, texture, and contraction by electrostimulation. ${ }^{9}$ However, at the anterior

5 anorectum, in addition to the above smooth and striated muscles, perineal muscles, urogenital

6 organs, and a fibromuscular mass and smooth muscle fibers, also known as the perineal body

7 and RUM, respectively, are complicatedly and three-dimensionally arranged. ${ }^{10,11}$ Furthermore,

8 the anatomical morphology or definitions of the perineal body and RUM are controversial, and

9 these terms are often confused by anatomists, urologists, and colorectal surgeons..$^{3,7,10-16}$ The

10 confusion seems to be brought about by the differences in anatomical methodology, such as

11 macroscopic observations during traditional anatomical dissection or microscopic observations

12 by two-dimensional and focal histological examination alone.

13 Large serial histological sections containing the entire anterior anorectum and three-

14 dimensional (3D) reconstruction would be required to correctly understand the spatial

15 relationship between striated and smooth muscles of the male anterior anorectum. This study

16 aimed to revisit the anatomical information of the male anterior anorectum for intersphincteric

17 resection and abdominoperineal resection with a focus on the spatial muscular morphology. 
2 Nine male cadavers (age range: 67-91 years) who had been donated to the Division of Anatomy

3 and Embryology at Ehime University Graduate School of Medicine were used for this study.

4 This study was conducted following the Act on Body Donation for Medical \& Dental Education

5 law of Japan and the provisions of the Declaration of Helsinki. The protocol for this study was

6 approved by the Ethical Committee of Ehime and Kyoto University.

7 Eight of nine cadavers were fixed by artery perfusion with $10 \%$ formalin, and the other cadaver

8 was fixed using Thiel's embalming method. ${ }^{17}$ The pelvises were cut off at the level of the

9 second sacral bone and the hip joint. These pelvises with the pelvic bones were placed into

10 boxes filled with $15 \%$ gelatin and hardened at $4{ }^{\circ} \mathrm{C}$ for $24-48$ hours to preserve the positional

11 relationship between the pelvic organs and pelvic bones. ${ }^{18,19}$ Then, the pelvises were frozen in

12 a freezer at $-20^{\circ} \mathrm{C}$ for 24 hours.

13 Nine pelvises embedded with gelatin were sectioned horizontally (3 pelvises), frontally (3), or

14 sagittally (3) at approximately 5-mm intervals by a slicer (NF-385K or NFK-360, Nantsune,

15 Osaka, Japan). The blade edge of this slicer was designed to have a sharp shape (not saw) to

16 minimize tissue loss. After all sections were photographed for macroscopic examinations by a

17 camera (EOS 7D MarkII, Canon, Tokyo, Japan), the specimens around the anterior anorectum 
1 were dissected for histological examinations to fit Super Mega Slim Cassettes (CellPath,

2 Powys, UK) that measured $75 \times 50 \times 5 \mathrm{~mm} .{ }^{12}$ The specimens were dehydrated in graded ethanol

3 and xylene, and embedded in paraffin blocks.

4 The specimens were serially sectioned at $10 \mu \mathrm{m}$. Every 25 th section was collected onto large

5 glass slides measuring $76 \times 52 \mathrm{~mm}$ and stained with Elastica van Gieson (EVG). Semi-serial

6 histological sections with an interval of $250 \mu \mathrm{m}$ were created. In addition, every 26 th and

$7 \quad 27$ th sections were collected onto glass slides. Some sections were stained using antibodies

8 against smooth muscle actin (Actin, Smooth Muscle Ab-1, Clone 1A4, Thermo Fisher

9 Scientific, Fremont, CA, USA) and skeletal muscle myosin (Myosin, Skeletal Muscle Ab-2,

10 Clone MYSN02, Thermo Fisher Scientific) to detect the smooth muscle and skeletal muscle

11 fibers, respectively. ${ }^{7}$ All slides were scanned as virtual slides using a slide scanner (BZX-700,

12 Keyence, Osaka, Japan).

13 Two series of semi-serial histological sections stained with EVG were used for the 3D

14 reconstruction. After manual registration of these series, smooth and striated muscles and

15 urogenital organs were segmented while referring to the immunohistochemical sections using

16 a 3D reconstruction software (Amira, Thermo Fisher Scientific). ${ }^{20}$

\section{Results}


Histological observations of horizontal sections

2 Figure 1 shows the horizontal histological sections of the anterior anorectum from the anal side

3 to the oral side. All slices of the semi-serial histological sections are shown in Supplemental

4 Digital Content 1.

$6 \quad$ External anal sphincter (EAS) level

7 At the EAS level (Fig. 1A-F), the EAS covered two smooth muscle layers of the rectal

8 muscularis propria: the circular muscle layer (CM) and the longitudinal muscle layer (LM).

9 Some smooth muscle fibers of the LM were inserted into the EAS near the upper limit of the

10 EAS (black arrowheads of Fig. 1E). The superficial transverse perineal muscle (STPM) was

11 identified as the striated muscle bundle running from the posterior area of the ischiocavernosus

12 muscle (ICM) toward the anterior part of the EAS. The thickness of this muscle had an inter-

13 individual morphological difference such as well or poorly developed types. Although the

14 STPM fibers terminated to the rectourethralis muscle (RUM) above the EAS on the lateral side

15 in only one of nine cadavers, the STPM fibers merged into the anterior part of the EAS in the

16 other cases, and the border of both muscles was inseparable. Similarly, the bulbospongiosus

17 muscle (BSM) was continuously attached to the EAS, and these striated muscles could not be 
1 separated. Thus, the EAS, STPM, and BSM were intertwined with each other and formed the

2 striated muscle complex (Fig. 1A-C). At the anterior part of the EAS, a fibromuscular mass

3 often referred to as the perineal body, where these perineal muscles converged to, as described

4 in anatomical atlases, ${ }^{21,22}$ was not identified.

\section{$6 \quad$ Puborectalis muscle (PRM) level}

7 At the PRM level (Fig. 1G-L), striated muscle fibers of the PRM sub-circumferentially covered

8 the rectal muscularis propria from the anterolateral to the lateral and posterior aspect. At the

9 anterior part, abundant smooth muscle fibers, which continued from the longitudinal muscle

10 (LM), extended anteriorly. In this study, the successive smooth muscle containing elastic and

11 collagen fibers was defined as the rectourethralis muscle (RUM). The RUM could not be

12 separated clearly from the LM.

13 At the lower PRM level (Fig. 1G-I), the RUM communicated with the corpus spongiosum

14 penis (CSP) and Cowper's gland (CG). Small striated muscle bundle extending laterally from

15 the posterior part of the CG was identified (black arrows of Fig. 1I). We believe that this muscle

16 corresponds to the deep transverse perineal muscle (DTPM) reported by previous studies. ${ }^{23,24}$

17 Although the thickness of the DTPM had an inter-individual morphological difference, it could 
1 not be identified as a thick sheet-like striated muscle spreading laterally just anterior to the

2 anorectum, as generally described in anatomical atlases. ${ }^{21,25}$ At the upper PRM level (Fig. 1J-

3 L), the RUM extended to the posterior area of the urethral rhabdosphincter (RS), which was a

4 striated muscle surrounding the urethra circularly.

5 At the anterolateral part, smooth muscle fibers from the LM were partially inserted into the

6 PRM, and a tight connection between the smooth and striated muscles was formed (black

7 arrowheads of Fig. $1 \mathrm{H}$ and $1 \mathrm{~K}$ ). This connection gradually became looser and changed into the

8 pelvic fascia, which covered the surface of the PRM at the prostate level (white arrowheads of

$9 \quad$ Fig. $1 \mathrm{M}$ and $1 \mathrm{~N})$.

11 Prostate level

12 At the prostatic apex level (Fig. 1M-O), the anterior extension of the rectourethralis muscle

13 (RUM) disappeared and the anterior border of the longitudinal muscle layer (LM) became clear.

14 Several nerve fibers, blood vessels, and adipose tissues were identified at the triangular area

15 surrounded by the rectum, puborectalis muscle (PRM), and prostate. This triangular area

16 corresponds to the neurovascular bundle (NVB) reported by Walsh. ${ }^{26}$ However, the mesorectal

17 fascia could not be clearly defined in adipose tissues at this level. Some nerves and blood 
1 vessels penetrated the RUM and supplied the rectal muscularis propria, while others descended

2 along the posterolateral and lateral sides of the urethral rhabdosphincter (RS) and supplied the

$3 \quad \mathrm{RS}$ and corpus spongiosum penis (CSP).

4

\section{$5 \quad$ Histological observations of sagittal sections}

6 Figure 2 shows the sagittal histological sections from the mid-sagittal to parasagittal sections.

7 All slices of semi-serial histological sections are shown in Supplemental Digital Content 2. The

8 urethra became closest to the rectal muscularis propria just inferior to the prostatic apex. At the

9 mid-sagittal section, the rectourethralis muscle (RUM) occupied the posterior-inferior area of

10 the urethra from the upper limit of the external anal sphincter (EAS) to the prostate apex (Fig.

11 2A-C). At the 3-mm parasagittal section, the RUM communicated with the urethral

12 rhabdosphincter (RS) and Cowper's gland (CG) (Fig. 2D-F). At the 8-mm parasagittal section,

13 the RUM fibers were partially inserted into the PRM (black arrowheads of Fig. $2 \mathrm{G}$ and $2 \mathrm{H}$ )

14 and changed into the pelvic fascia of the PRM (white arrowheads of Fig. $2 \mathrm{G}$ and $2 \mathrm{H}$ ).

$3 D$ reconstruction

17 Figures 3 and 4 show the left anterior oblique view and transanal view of the 3D reconstruction 
1 images of the anterior anorectum, respectively. The multi-angle views of the segmented

2 histological sections and 3D images are shown in Supplemental Digital Content 3.

3 These 3D images can be used to visualize the spatial relationship between the smooth (blue)

4 and striated (red) muscles, especially the three-dimensional extension of the rectourethralis

5 muscle (RUM). The RUM, which was inseparable from the longitudinal muscle (LM), filled

6 the space surrounded by the striated muscle complex, which consisted of the external anal

7 sphincter (EAS), bulbospongiosus muscle (BSM), and superficial transverse perineal muscle

8 (STPM), the urogenital organs of the prostate and urethra, and the bilateral slings of the

9 puborectalis muscle (PRM) (Figs. 3B, 4C, and 4D). After division of the striated muscle

10 complex in abdominoperineal resection or the separation at the intersphincteric plane between

11 the EAS and LM in intersphincteric resection at the EAS level, both dissection planes always

12 encounter the RUM at the PRM level. Since the urethra becomes close to the anorectum at the

13 prostatic apex level as the 3D image shows (Figs. 3A, 3B, 4C, and 4D), surgeons have to be

14 careful to avoid urethra injury during the division of the RUM. 


\section{Discussion}

2 Large semi-serial histological sections and the 3D reconstruction images precisely clarified the

3 spatial muscular morphology of the male anterior anorectum. The detailed descriptions of the

4 relationship between striated and smooth muscles at each level will enable more accurate step-

5 by-step anterior dissection in intersphincteric resection and abdominoperineal resection,

6 especially with the transanal approach. Moreover, the 3D image can be used to visualize the

$73 \mathrm{D}$ anatomical structure hidden behind the dissection plane by the multi-angle views and

8 transparent organs, which is informative for surgeons so they can avoid unexpected

9 complications.

11 Anatomical structure of the anterior anorectum at the external anal sphincter (EAS) level

12 The anterior structure of the male EAS has been generally described as striated muscle fibers

13 of the EAS, bulbospongiosus muscle (BSM), and superficial transverse perineal muscle

14 (STPM) that converge to the perineal body, which comprises the fibromuscular mass in

15 anatomical atlases. ${ }^{21,22}$ However, such a fibromuscular mass could not be identified anterior to

16 the EAS in this study. Instead, these striated muscle fibers were intertwined with each other

17 and formed the striated muscle complex (Figs. 1A, 1C, and 4A). This finding is important 
1 knowledge for the anterior dissection of abdominoperineal resection. Surgeons have to keep in

2 mind that there is no clear border between the STPM, BSM, and EAS. The STPM fibers, which

3 run transversally from the posterior ischiocavernosus muscle to the anterior EAS, should be

4 identified at both anterolateral parts of the EAS. Subsequently, the striated muscle complex is

5 divided while confirming the STPM fibers that have already been exposed at both anterolateral

6 parts.

7 At the anterior part of the upper EAS level, the smooth muscle fibers of the longitudinal muscle

8 (LM) were partially inserted into the EAS (arrowheads of Figs. 1E, 2E, and Fig. 4B) and

9 communicated cephalad with the rectourethralis muscle (RUM). This finding indicates that the

10 separation at the intersphincteric plane gradually becomes difficult as the upper EAS level is

11 approached. This connection should be sharply divided at the inside of the EAS, confirming

12 the contraction of the EAS by electrostimulation; subsequently, the RUM at the puborectalis

13 muscle (PRM) level will be encountered in the dissection plane.

Anatomical structure of the anterior anorectum at the puborectalis muscle (PRM) level

16 In this study, we defined wide anterior extension of the smooth muscle, which continued from

17 the longitudinal muscle (LM), as the rectourethralis muscle (RUM) (Figs. 1H, 1K, 2B, 2E, 3B, 
$14 \mathrm{C}$, and 4D). The RUM has been generally described as smooth muscles that interposed

2 between the anorectum and urethra. ${ }^{7,14,27}$ The term RUM appears to be mostly established

3 among urologists as a part where rectal wall injury tends to occur during perineal radical

4 prostatectomy. ${ }^{13,14,16,27-29}$ Similarly, in intersphincteric resection or abdominoperineal resection,

5 there are always risks of rectal or urethral injury during the division of the RUM due to the

6 unclear border between the urethra and anorectum.

7 On the contrary, this part is also often called the perineal body, instead of the RUM. ${ }^{3,10-12}$

8 Therefore, the definition of the perineal body is confusing and controversial, including the

9 anterior part of the EAS as described above, ${ }^{13-16,27,28,30}$ the area between the urethra and

10 anorectum, ${ }^{10}$ or the whole area below the prostate and perineal skin. ${ }^{3,11,12}$ In this study, the

11 anterior anorectum consists of striated and smooth muscles, and a specific structure

12 corresponding to the perineal body could not be distinguishable. Instead, Muro et al. described

13 the perineal body as a region between the rectum, anal canal, and urogenital organs, which

14 seemed to be reasonable to prevent terminological confusion. ${ }^{31}$

15 At the anterolateral part, the smooth muscle fibers from the LM were inserted into the

16 puborectalis muscle (PRM) of the levator ani muscle (black arrowheads of Figs. 1H, 1K and

17 2H). The connection between the levator ani muscle and LM identified not only anterolaterally 
1 but sub-circumferentially from the anterolateral part to the posterior part of the anorectum. ${ }^{6,32}$

2 Additionally, especially at the posterior part, the thick smooth muscle bundle, the so-called

3 anococcygeal ligament or ileorectococcygeus muscle, extended from the LM to the superior

4 aspect of the coccyx..$^{8,33,34}$ Shafik described these connections as the "hiatal ligament," which

5 circumferentially filled the gap between the levator ani muscle and the anorectum or the

6 peripheral organs. ${ }^{35}$ That is, the anorectum is sub-circumferentially fixed to the levator ani

7 muscle via the connection between the LM and the levator ani muscle, and it is connected to

8 urogenital organs via the RUM at the anterior part and to the coccyx via the anococcygeal

9 ligament or ileorectococcygeus muscle at the posterior part. These connections are the entrance

10 into the intersphincteric plane, and must be sharply divided when intersphincteric resection is

11 performed.

12 During the anterior dissection of abdominoperineal resection at the PRM level, at first, at the

13 anterolateral part, bilateral slings of the PRM are divided. There is no clear anatomical

14 landmark to divide the PRM. We believe that the division behind the STMP level would be

15 appropriate for standard abdominoperineal resection unless tumor involvement is suspected.

16 On the other hand, during intersphincteric resection, the tight connection between the LM and

17 PRM is sharply divided along the inside of the PRM, while confirming the contraction of the 
1 PRM by electrostimulation. However, since this connection gradually changes into the pelvic

2 fascia of the PRM (white arrowheads of Figs. $1 \mathrm{M}, 1 \mathrm{~N}, 2 \mathrm{G}$, and $2 \mathrm{H}$ ), too wide separation along

3 the RPM would lead to the wide exposure of the lateral aspect of the neurovascular bundle and

4 the prostate. Contrarily, deviation of the dissection plane into the longitudinal muscle may lead

5 to rectal perforation. In both abdominoperineal resection and intersphincteric resection, there

6 is no anatomical border around the RUM at the anterior part, where the urethra becomes closest

7 to the anorectum. Surgeons should keep in mind that division of the RUM always has the

8 potential risk of urethral injury or rectal perforation. Palpation of a urinary catheter, ${ }^{36}$

9 visualization of the urethra using a lighting stent, ${ }^{37-39}$ and observation of the dissection plane

10 from the transabdominal and the transanal teams may be useful to avoid these complications.

11 This study had two limitations. First, most cadavers used in this study were elderly with

12 degenerative changes caused by aging. Second, the study had a small sample size. Despite

13 these limitations, we believe that large semi-serial histological sections are comparable to

14 magnetic resonance imaging (MRI) obtained from patients with rectal cancer. In the future, we

15 would like to create a precise 3D anatomical model of the pelvic floor that considers individual

16 variations by using the patient's MRI for reconstruction, and to establish a safer surgical

17 technique for intersphincteric resection and abdominoperineal resection by using this 3D model 
1 for surgical planning and image-guided navigation. 


\section{Conclusions}

2 Large semi-serial histological sections and 3D reconstruction enabled precise visualization of

3 the spatial relationship between smooth and striated muscles of the male anterior anorectum.

4 Two regions without a clear anatomical border were elucidated: (i) the anterior region of the

5 external anal sphincter(EAS), where the striated fibers from the EAS, bulbospongiosus muscle

6 (BSM), and superficial transverse perineal muscle (STPM) were intertwined, and (ii) the

7 rectourethralis muscle (RUM), where the smooth muscle fibers from the longitudinal muscle

8 continuously extended to the posteroinferior area of the urethra, which became closest to the

9 anorectum at the prostatic apex level. During the division of the RUM at the prostatic apex

10 level, surgeons have to be careful to avoid urethral or rectal injury. Furthermore, tight

11 connection between the striated and smooth muscles was identified at two regions: (i) the

12 anterior part of the upper EAS level, and (ii) anterolateral part of the puborectalis muscle level.

13 We believe that these detailed anatomical findings will contribute to more appropriate anterior

14 dissection during intersphincteric resection and abdominoperineal resection, especially with

15 the transanal approach, which can magnify the muscle fiber direction and the contraction of

16 striated muscle by electrostimulation. 


\section{Acknowledgments}

2 The authors thank Takuya Doihara from Ehime University for sampling the cadaver specimens,

3 Takaaki Matsumoto and Atsushi Miyawaki from Nantsune Corporation for providing technical

4 support for sectioning the specimens, Itsuro Kamimura from Maxnet Corporation for providing

5 technical support for three-dimensional reconstructions, Osamu Takahashi from Keyence

6 Corporation for providing technical support for scanning the serial histological sections, and

7 Editage (www.editage.jp) for English language editing. This work was supported by the Japan

8 Society for the Promotion of Science (KAKENHI numbers: 16H05399 and 16K10538). 


\section{$1 \quad$ References}

2 1. den Dulk M, Putter H, Collette L, et al. The abdominoperineal resection itself is associated

3 with an adverse outcome: the European experience based on a pooled analysis of five

4 European randomised clinical trials on rectal cancer. Eur J Cancer. 2009;45:1175-1183.

5 2. Fleshman J, Branda ME, Sargent DJ, et al. Disease-free survival and local recurrence for

6 laparoscopic resection compared with open resection of stage II to III rectal cancer:

7 follow-up results of the ACOSOG Z6051 randomized controlled trial. Ann Surg.

$8 \quad 2019 ; 269: 589-595$.

9 3. Stelzner S, Holm T, Moran BJ, et al. Deep pelvic anatomy revisited for a description of

10 crucial steps in extralevator abdominoperineal excision for rectal cancer. Dis Colon

$11 \quad$ Rectum. 2011;54:947-957.

12 4. Penna M, Hompes R, Arnold S, et al. Incidence and risk factors for anastomotic failure in 1594 patients treated by transanal total mesorectal excision: results from the international

TaTME Registry. Ann Surg. 2019;269:700-711.

15 5. Rouanet P, Mourregot A, Azar CC, et al. Transanal endoscopic proctectomy: an innovative 
1 6. Tsukada Y, Ito M, Watanabe K, et al. Topographic anatomy of the anal sphincter complex and levator ani muscle as it relates to intersphincteric resection for very low rectal disease. Dis Colon Rectum. 2016;59:426-433.

4 7. Nakajima Y, Muro S, Nasu H, Harada M, Yamaguchi K, Akita K. Morphology of the region anterior to the anal canal in males: visualization of the anterior bundle of the longitudinal muscle by transanal ultrasonography. Surg Radiol Anat. 2017;39:967-973.

8. Muro S, Yamaguchi K, Nakajima Y, et al. Dynamic intersection of the longitudinal muscle and external anal sphincter in the layered structure of the anal canal posterior wall. Surg Radiol Anat. 2014;36:551-559.

9. Batista-Lima FJ, Gadelha KKL, Oliveira DM, Vasconcelos TB, Brito TS, Magalhaes PJC.

A simple laboratory exercise with rat isolated esophagus and stomach fundus to reveal functional differences between striated and smooth muscle cells. Adv Physiol Educ. 2017;41:291-297.

10. Oh C, Kark AE. Anatomy of the perineal body. Dis Colon Rectum. 1973;16:444-454.

15 11. Wu Y, Dabhoiwala NF, Hagoort J, et al. 3D topography of the young adult anal sphincter complex reconstructed from undeformed serial anatomical sections. PLoS One. 
1 12. Kraima AC, West NP, Treanor D, et al. The anatomy of the perineal body in relation to abdominoperineal excision for low rectal cancer. Colorectal Dis. 2016;18:688-695.

3 13. Zhai LD, Liu J, Li YS, Ma QT, Yin P. The male rectourethralis and deep transverse perineal muscles and their relationship to adjacent structures examined with successive slices of celloidin-embedded pelvic viscera. Eur Urol. 2011;59:415-421.

14. Porzionato A, Macchi V, Gardi M, Parenti A, Caro RD. Histotopographic study of the rectourethralis muscle. Clin Anat. 2005;18:510-517.

15. Uchimoto K, Murakami G, Kinugasa Y, Arakawa T, Matsubara A, Nakajima Y. Rectourethralis muscle and pitfalls of anterior perineal dissection in abdominoperineal resection and intersphincteric resection for rectal cancer. Anat Sci Int. 2007;82:8-15.

16. Brooks JD, Eggener SE, Chao WM. Anatomy of the rectourethralis muscle. Eur Urol. 2002;41:94-100.

17. Thiel W. The preservation of the whole corpse with natural color. Ann Anat. 1992;174:185-195. improved serially sectioned images of the entire body. IEEE Trans Med Imaging. 
1 19. Spitzer V, Ackerman MJ, Scherzinger AL, Whitlock D. The visible human male: a technical report. J Am Med Inform Assoc. 1996;3:118-130.

20. Takashima Y, Terada M, Kawabata M, Suzuki A. Dynamic three-dimensional morphogenesis of intrahepatic bile ducts in mouse liver development. Hepatology. 2015;61:1003-1011.

21. Netter FH. Atlas of Human Anatomy. 6th ed. Philadelphia: Saunders Elsevier; 2014.

22. Kopf-Maier P, ed. Wolf-Heidegger's Atlas of Human Anatomy. 6th ed. Basel: Karger; 2005.

23. Murakami G, Nakajima F, Sato TJ, Tsugane MH, Taguchi K, Tsukamoto T. Individual variations in aging of the male urethral rhabdosphincter in Japanese. Clin Anat. $2002 ; 15: 241-252$.

24. Nakajima F, Takenaka A, Uchiyama E, Hata F, Suzuki D, Murakami G. Macroscopic and histotopographic study of the deep transverse perineal muscle (musculus transversus perinei profundus) in elderly Japanese. Ann Anat. 2007;189:65-74.

25. Agur AMR, Dalley AF. Grant's Atlas of Anatomy. 12th ed. Baltimore: Williams \& Wilkins; 2009.

26. Walsh PC. Anatomical studies of the neurovascular bundle and cavernosal nerves. $J$ Urol. 
1 27. Soga H, Takenaka A, Murakami G, Fujisawa M. Topographical relationship between

urethral rhabdosphincter and rectourethralis muscle: a better understanding of the apical dissection and the posterior stitches in radical prostatectomy. Int J Urol. 2008;15:729-732.

28. Myers RP, Cahill DR, Devine RM, King BF. Anatomy of radical prostatectomy as defined by magnetic resonance imaging. J Urol. 1998;159:2148-2158.

29. Matsubara A, Murakami G, Arakawa T, et al. Topographic anatomy of the male perineal structures with special reference to perineal approaches for radical prostatectomy. Int $J$ Urol. 2003;10:141-148.

30. Aigner F, Zbar AP, Ludwikowski B, Kreczy A, Kovacs P, Fritsch H. The rectogenital septum: morphology, function, and clinical relevance. Dis Colon Rectum. 2004;47:131140.

31. Muro S, Tsukada Y, Harada M, Ito M, Akita K. Spatial distribution of smooth muscle tissue in the male pelvic floor with special reference to the lateral extent of the rectourethralis muscle: Application to prostatectomy and proctectomy. Clin Anat. 2018;31:1167-1176.

32. Arakawa T, Murakami G, Nakajima F, et al. Morphologies of the interfaces between the levator ani muscle and pelvic viscera, with special reference to muscle insertion into the 
anorectum in elderly Japanese. Anat Sci Int. 2004;79:72-81.

2 33. Courtney H. Anatomy of the pelvic diaphragm and anorectal musculature as related to sphincter preservation in anorectal surgery. Am J Surg. 1950;79:155-173.

4 34. Kinugasa Y, Arakawa T, Abe S, et al. Anatomical reevaluation of the anococcygeal ligament and its surgical relevance. Dis Colon Rectum. 2011;54:232-237.

35. Shafik A. Levator ani muscle: new physioanatomical aspects and role in the micturition mechanism. World J Urol. 1999;17:266-273.

36. Adamina M, Buchs NC, Penna M, Hompes R; St.Gallen Colorectal Consensus Expert Group. St.Gallen consensus on safe implementation of transanal total mesorectal excision. Surg Endosc. 2018;32:1091-1103. lighted stent as a method for identifying the urethra in male patients undergoing transanal total mesorectal excision: a video demonstration. Tech Coloproctol. 2015;19:375.

38. Okada T, Kawada K, Nakamura T, et al. A cadaveric demonstration of visualization of the urethra using a lighted stent during transanal intersphincteric resection. International Cancer Conference Journal. 2018;7:77-80.

17 39. Kawada K, Hida K, Yoshitomi M, Sakai Y. Use of a lighted stent to identify the urethra 


\section{Figure Legends}

\section{Figure 1. Horizontal histological sections}

3 Horizontal histological sections of a 73-year-old male cadaver showing Elastica van Gieson

4 (EVG), anti-smooth muscle, and anti-striated muscle staining from the anal side to oral side:

5 lower (A-C) and upper (D-F) EAS level, lower (G-I) and upper (J-L) PRM level, and

6 prostatic apex level (M-O). The striated muscle fibers (black dashed arrows of A) of the

7 BSM, STPM, and EAS are intertwined and form the striated muscle complex. The RUM,

8 which comprises smooth muscle fibers that continue from the LM, extends to the CG, CSP

$9(\mathrm{H})$, and RS (K) and contains the DTPM (black arrows of I) of the striated muscle. Smooth

10 muscle fibers are inserted into the striated muscle (black arrowheads of $\mathrm{E}, \mathrm{H}$ and $\mathrm{K}$ ), which

11 change into the pelvic fascia (white arrowheads of $\mathrm{M}$ and $\mathrm{N}$ ).

12 BSM=bulbospongiosus muscle; $\mathrm{CG}=$ Cowper's gland; $\mathrm{CM}=$ circular muscle layer;

$13 \mathrm{CSP}=$ corpus spongiosum penis; DTPM=deep transverse perineal muscle; $\mathrm{EAS}=$ external anal

14 sphincter; ICM=ischiocavernosus muscle; $\mathrm{LM}=$ longitudinal muscle; $\mathrm{NVB}=$ neurovascular

15 bundle; $\mathrm{Pr}=$ prostate; $\mathrm{PRM=}$ =puborectalis muscle; RS=rhabdosphincter; RUM=rectourethralis

16 muscle; STPM=superficial transverse perineal muscle; $\mathrm{Ur}=$ urethra 


\section{Figure 2. Sagittal histological sections}

2 Sagittal histological sections of a 67-year-old male cadaver showing Elastica van Gieson

3 (EVG), anti-smooth muscle, and anti-striated muscle staining: mid-sagittal sections (A-C), 3-

$4 \mathrm{~mm}(\mathrm{D}-\mathrm{F})$ and $8-\mathrm{mm}(\mathrm{G}-\mathrm{I})$ parasagittal sections. The urethra becomes closest to the rectal

5 muscularis propria just inferior to the prostatic apex (A-C). The RUM extends to the urethra

6 and CSP (B), RS, and CG (E). Black arrows indicate the striated muscle of the DTPM (F).

7 Smooth muscle fibers are inserted into the striated muscle (black arrowheads of D, E, G, and

$8 \mathrm{H}$ ), and change into the pelvic fascia (white arrowheads of $\mathrm{G}$ and $\mathrm{H}$ ).

9 BSM=bulbospongiosus muscle; $\mathrm{CG}=$ Cowper's gland; $\mathrm{CM}=$ circular muscle layer;

$10 \mathrm{CSP}=$ corpus spongiosum penis; $\mathrm{DTPM}=\mathrm{deep}$ transverse perineal muscle; $\mathrm{EAS}=$ external anal

11 sphincter; $\mathrm{LM}=$ longitudinal muscle layer; $\mathrm{NVB}=$ neurovascular bundle; $\mathrm{Pr}=$ prostate;

$12 \mathrm{PRM}=$ puborectalis muscle; $\mathrm{RS}=$ rhabdosphincter; $\mathrm{RUM}=$ rectourethralis muscle; $\mathrm{Ur}=$ urethra

14 Figure 3. Left anterior oblique view of the 3D reconstruction image

15 The 3D image was reconstructed from horizontal semi-serial histological sections in Figure 1.

16 (A) Peripheral 3D structure of the RUM. (B) Successive smooth muscle of the LM and RUM

17 to A. (C) Striated muscle of the PRM and ICM to B. 
$1 \mathrm{BSM}=$ bulbospongiosus muscle; $\mathrm{CG}=$ Cowper's gland; $\mathrm{CM}=$ circular muscle; $\mathrm{CSP}=$ corpus

2 spongiosum penis; DTPM=deep transverse perineal muscle; EAS=external anal sphincter;

3 ICM=ischiocavernosus muscle; $\mathrm{LM}=$ longitudinal muscle layer; $\mathrm{Pr}=$ prostate;

$4 \quad \mathrm{PRM}=$ puborectalis muscle; $\mathrm{RS}=$ rhabdosphincter; $\mathrm{RUM}=$ rectourethralis muscle; $\mathrm{Ur}=$ urethra

6 Figure 4. Transanal view of the 3D reconstruction image of Figure 3

7 Transanal view for the anterior dissection of intersphincteric resection or abdominoperineal

8 resection with the transanal approach. (A and B) Lower and upper EAS levels, respectively.

9 (C and D) Lower and upper PRM levels, respectively. (E) Prostatic apex level. The STPM

10 (black dashed arrows of A) merges into the anterior EAS. The smooth muscle fibers (RUM)

11 continue from the LM, extend widely to the anterior area, and are partially inserted into the

12 PRM at the anterolateral part (C and D).

$13 \mathrm{BSM}=$ bulbospongiosus muscle; $\mathrm{CG}=$ Cowper's gland; $\mathrm{CM}=$ circular muscle; $\mathrm{CSP}=$ corpus

14 spongiosum penis; DTPM=deep transverse perineal muscle; EAS=external anal sphincter;

$15 \mathrm{ICM}=$ ischiocavernosus muscle; $\mathrm{LM}=$ longitudinal muscle; $\mathrm{NVB}=$ neurovascular bundle;

$16 \mathrm{Pr}=$ prostate; $\mathrm{PRM}=$ puborectalis muscle; $\mathrm{RS}=$ rhabdosphincter; $\mathrm{RUM}=$ rectourethralis muscle;

17 STPM=superficial transverse perineal muscle; $\mathrm{Ur}=$ urethra 

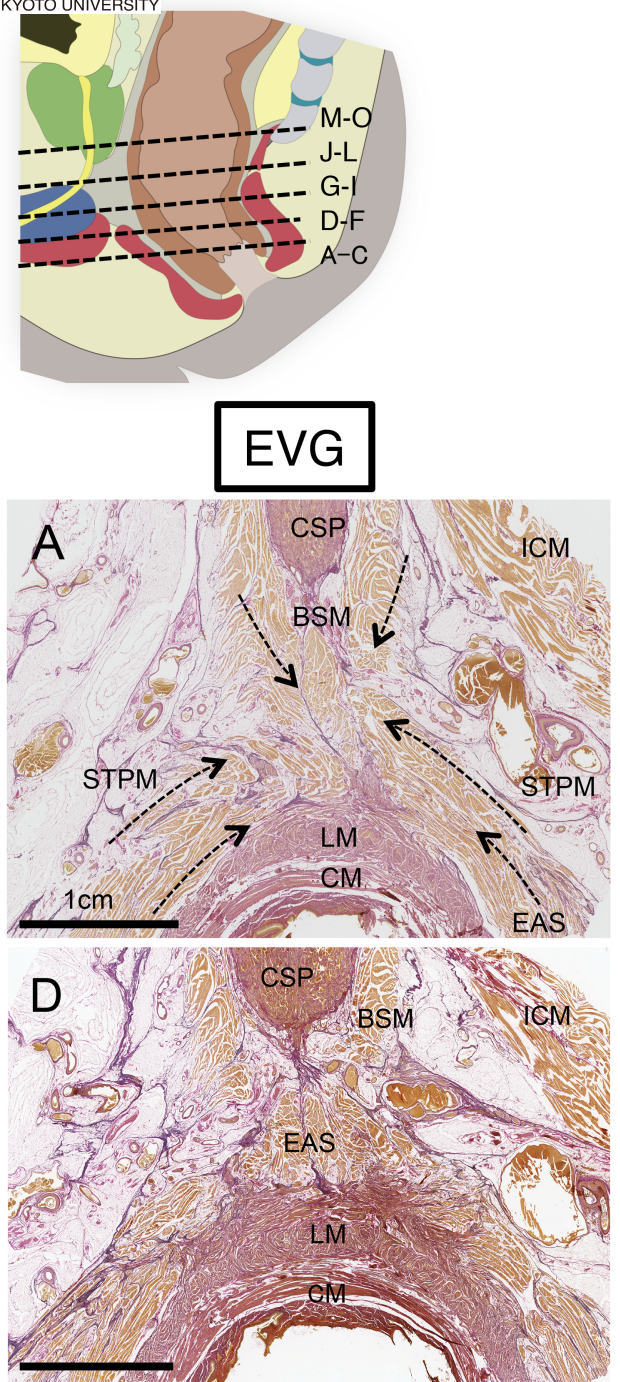

\section{G}
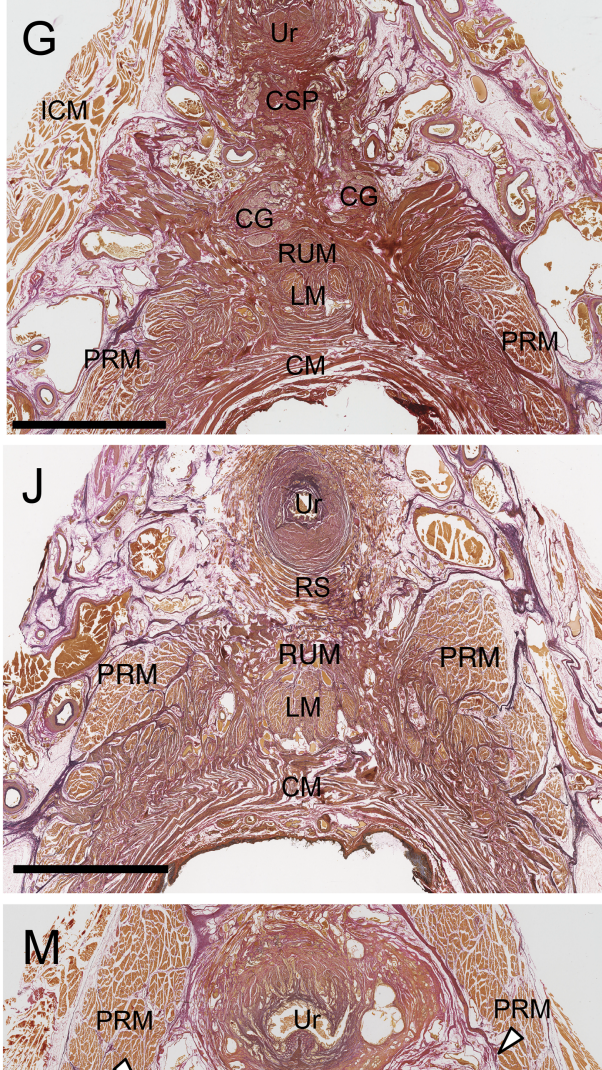

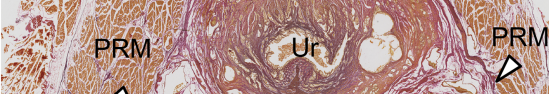

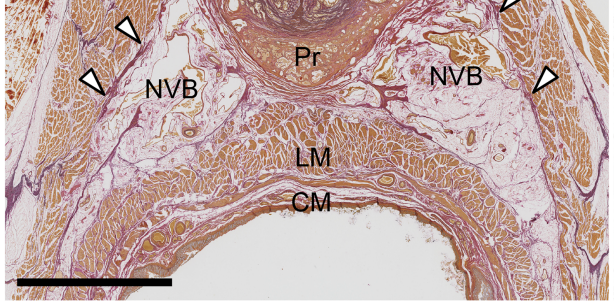

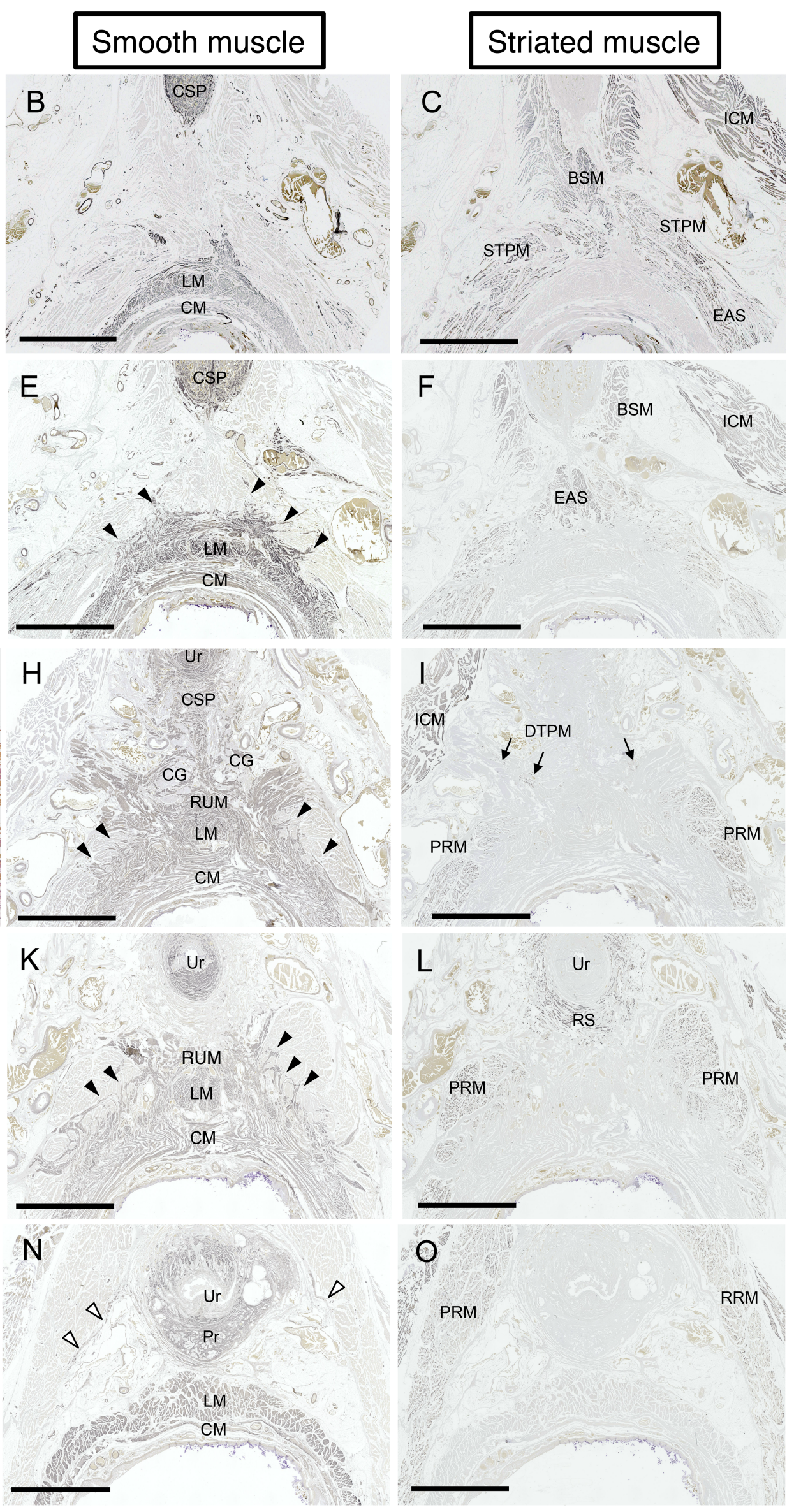




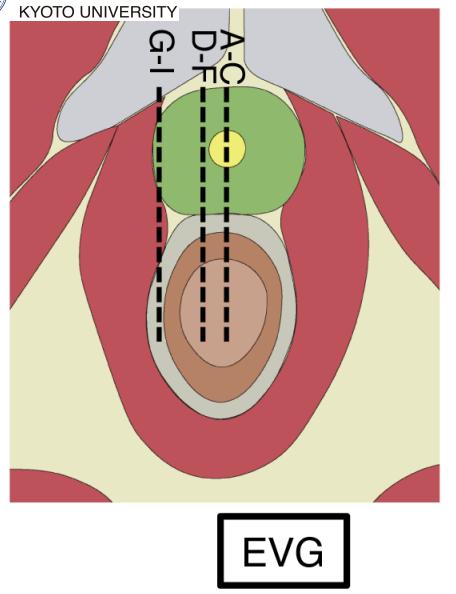

\section{Striated muscle}

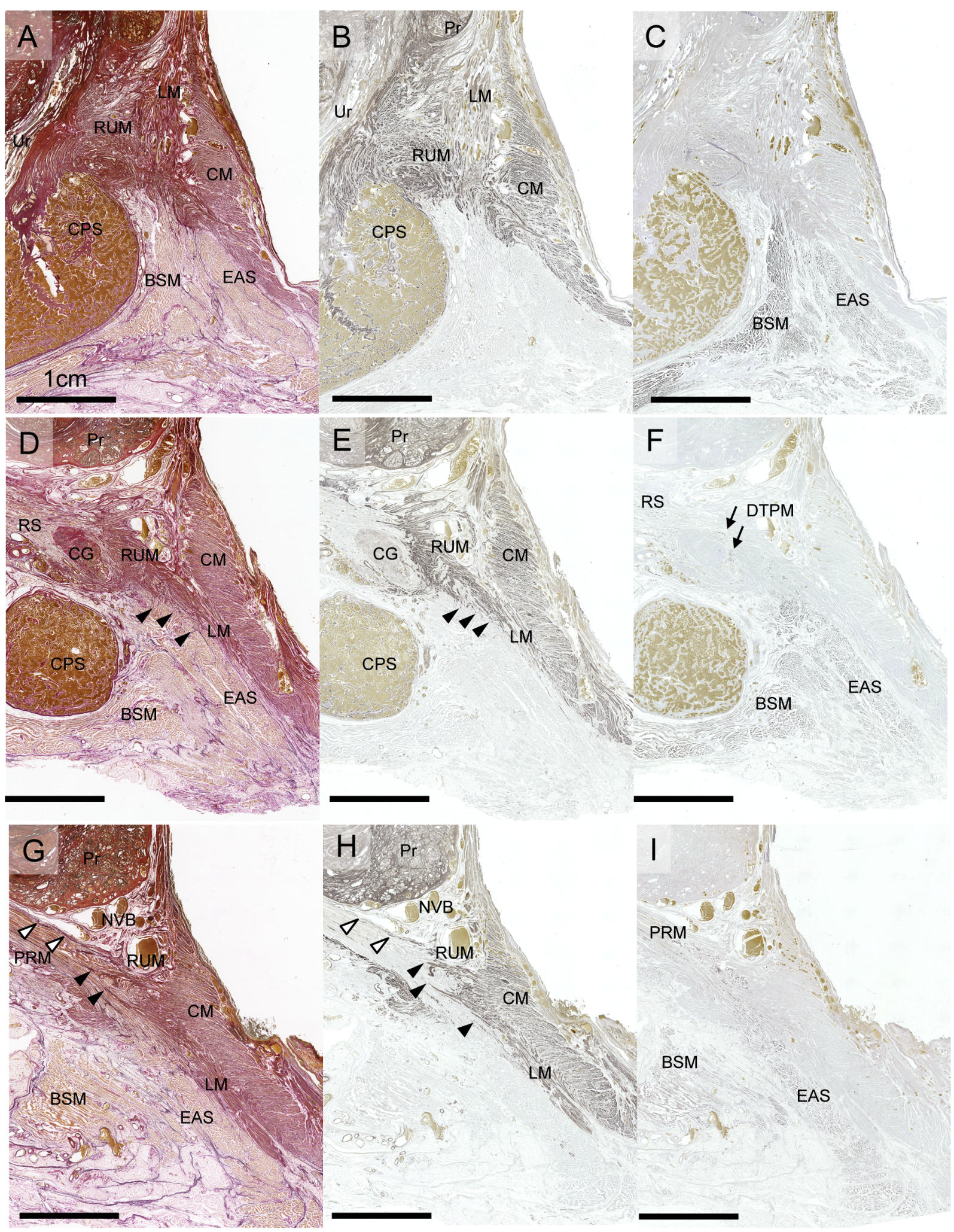


(2) 京都大学
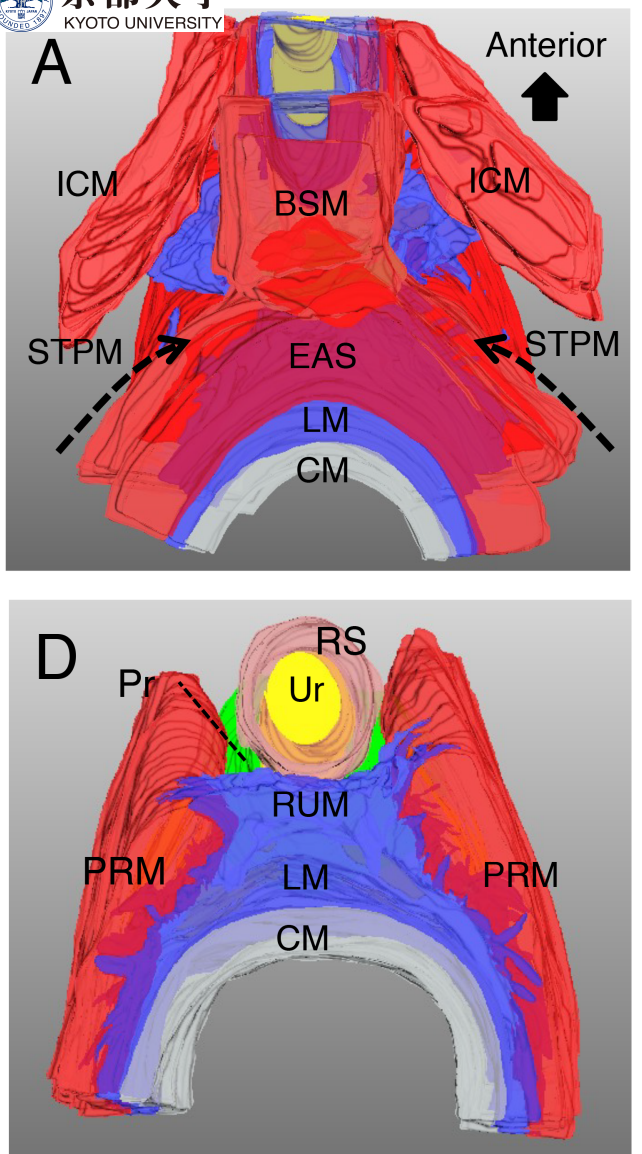

Kyoto University Research Information Repository
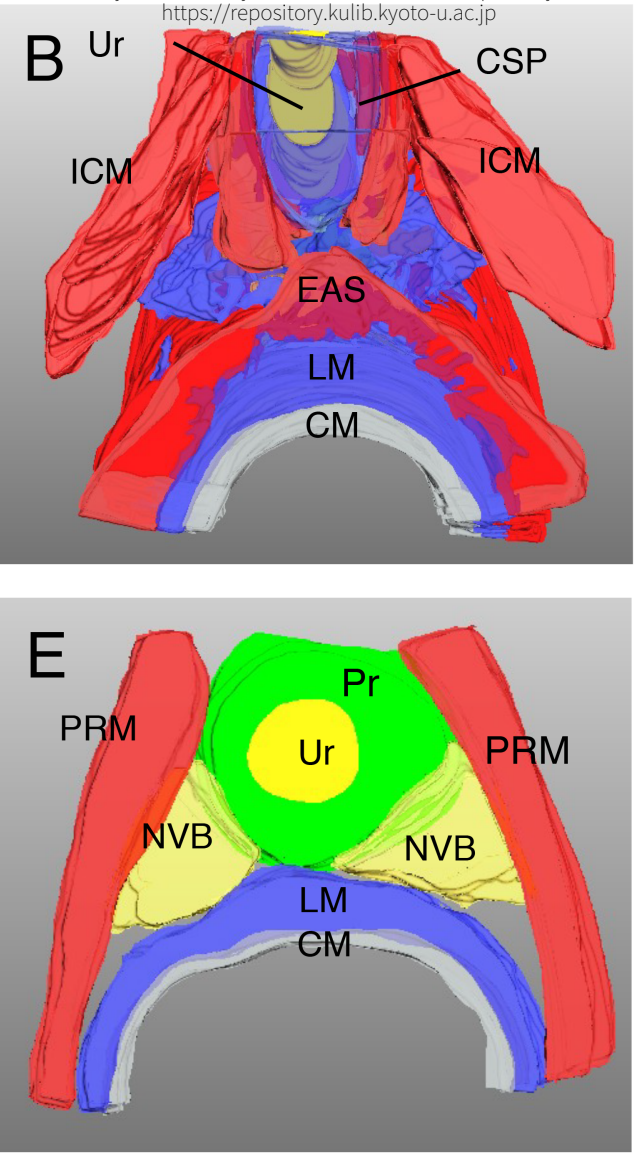

KURENAII 㣔
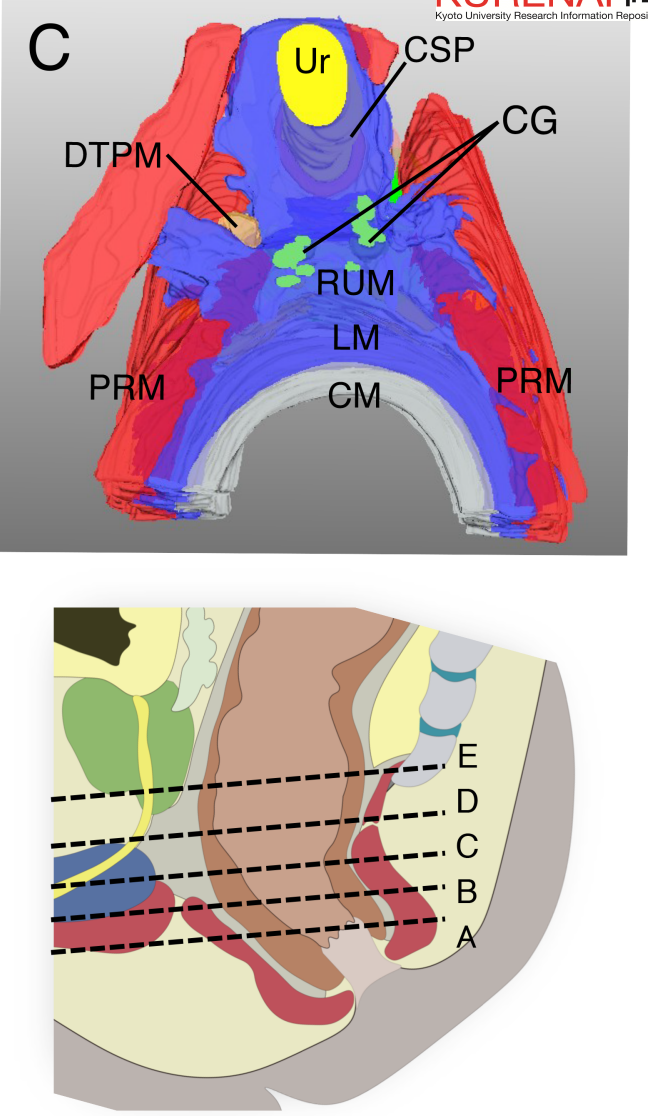

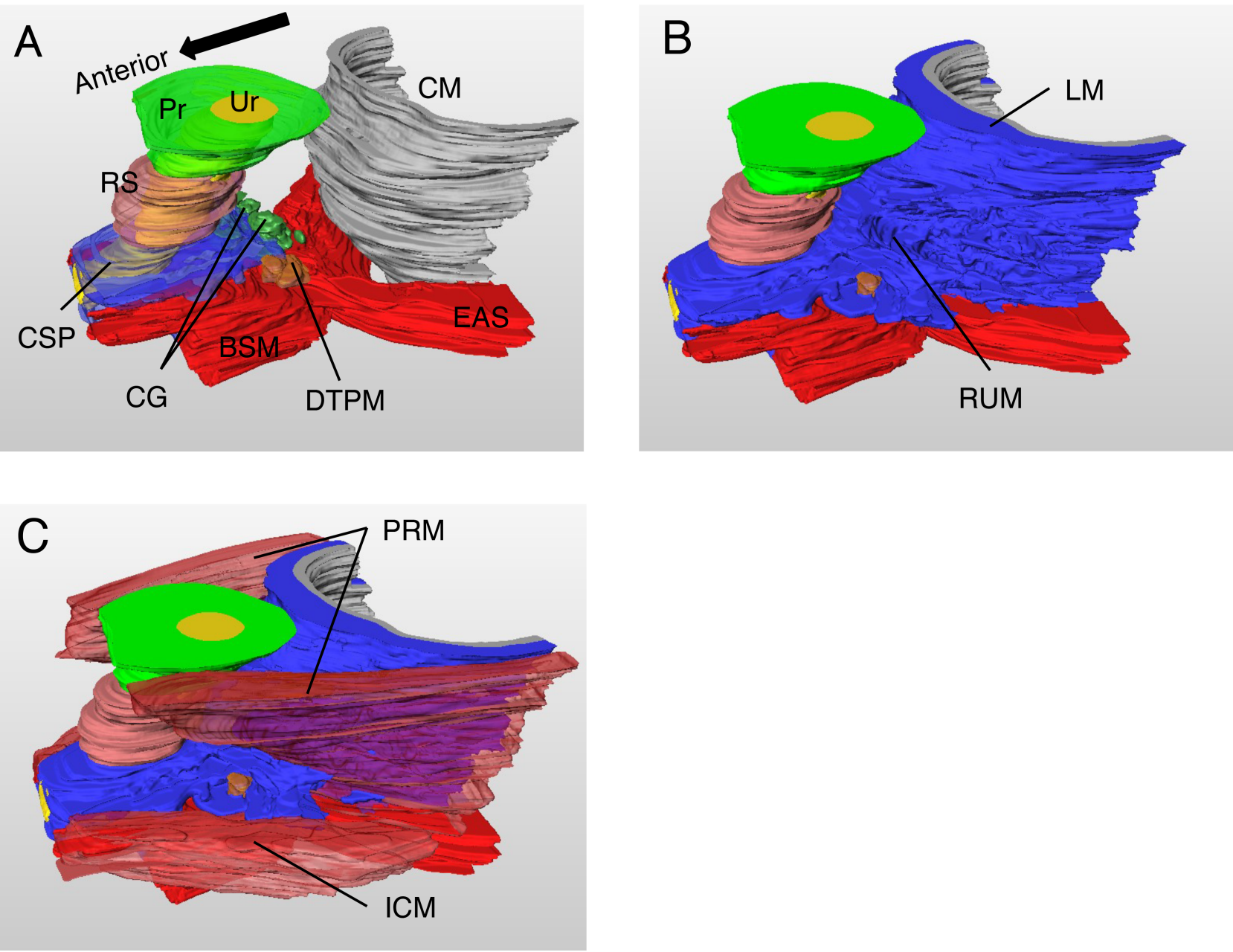